ARTICLE

Received 5 Sep 2012 | Accepted 1 May 2013 | Published 12 Jun $2013 \quad$ DOl: 10.1038/ncomms2965 OPEN

\title{
Integrating DNA strand-displacement circuitry with DNA tile self-assembly
}

David Yu Zhang ${ }^{1, *, \dagger}$, Rizal F. Hariadi ${ }^{2, \star}, \dagger$, Harry M.T. Choi ${ }^{3}$ \& Erik Winfree ${ }^{1,3,4}$

DNA nanotechnology has emerged as a reliable and programmable way of controlling matter at the nanoscale through the specificity of Watson-Crick base pairing, allowing both complex self-assembled structures with nanometer precision and complex reaction networks implementing digital and analog behaviors. Here we show how two well-developed frameworks, DNA tile self-assembly and DNA strand-displacement circuits, can be systematically integrated to provide programmable kinetic control of self-assembly. We demonstrate the triggered and catalytic isothermal self-assembly of DNA nanotubes over $10 \mu \mathrm{m}$ long from precursor DNA double-crossover tiles activated by an upstream DNA catalyst network. Integrating more sophisticated control circuits and tile systems could enable precise spatial and temporal organization of dynamic molecular structures.

\footnotetext{
${ }^{1}$ Department of Computation and Neural Systems, California Institute of Technology, Pasadena, California, USA. ${ }^{2}$ Department of Applied Physics, California Institute of Technology, Pasadena, California, USA. ${ }^{3}$ Department of Bioengineering, California Institute of Technology, Pasadena, California, USA. ${ }^{4}$ Department of Computer Science, California Institute of Technology, Pasadena, California, USA. * These authors contributed equally to this work. $\dagger$ Present addresses: Department of Bioengineering, Rice University, Houston, Texas, USA (D.Y.Z.); Department of Cell and Developmental Biology, University of Michigan, Ann Arbor, Michigan, USA (R.F.H.). Correspondence and requests for materials should be addressed to D.Y.Z. (email: dyz1@rice.edu) or to E.W. (email: winfree@caltech.edu).
} 
$\mathrm{B}$ iological cells are self-organized information-based molecular systems that acquire material and information, make molecules and decisions, and take actions that regulate their internal functions and their interactions with the environment ${ }^{1}$. The mechanisms underlying such behaviors involve an interplay between biochemical control circuitry and self-assembly processes. For example, in contrast to viruses that self-assemble from constitutively expressed proteins ${ }^{2}$, pathogenic bacteria often actively regulate construction of organelles such as flagella to escape immune responses, conserve resources and improve assembly yield ${ }^{3}$. Even more sophisticated examples exist in eukaryotic cells, where biochemical circuits modulate microtubule growth for tasks such as separating chromosomes during mitosis ${ }^{4}$, determining cell shape and polarity ${ }^{5,6}$, probing the environment with filopodia ${ }^{7}$, and directing cellular motility with lamellipodia ${ }^{7}$. These biological systems illustrate the potential of regulated self-assembly for complex molecular construction and dynamic behavior; it follows that engineering regulated self-assembly is a promising approach for expanding the capabilities of molecular nanotechnology ${ }^{8}$ and could facilitate the construction of synthetic cells $s^{9,10}$ and nanoscale robotic systems ${ }^{11-13}$.

Structural DNA nanotechnology offers a promising approach to constructing nanoscale components ${ }^{14}$. The specificity of Watson-Crick hybridization of oligonucleotides and the geometric predictability of the double helix enables precise rational design of primary sequence to achieve reliable selfassembly of target molecular structures. Often synthetic oligonucleotides are designed so as to hierarchically selfassemble first into nanometer-scale building blocks, which subsequently assemble into micron- or even millimeter-scale structures $^{15,16}$. Cooperative self-assembly of building blocks can result in complex 'algorithmically' patterned structures that construct different objects based on information present in a 'seed' structure, conferring a degree of self-regulation to the systematically programmed self-assembly reactions ${ }^{17-19}$.

Thermal annealing is frequently used as an easy and robust way to coordinate the desired sequence of self-asembly events in structural DNA nanotechnology. In contrast, self-assembled biological components grow, reconfigure and shrink isothermally in response to cellular or environmental signals. Conditional and isothermal assembly of DNA structures may also be desirable for applications in biomedical technology and materials science, for example. While isothermal tile-based self-assembly has been demonstrated ${ }^{20,21}$, controlling desired and undesired nucleation ${ }^{19,22}$ can make the temporal and spatial regulation of these processes difficult.

In dynamic DNA nanotechnology ${ }^{23}$, isothermal DNA stranddisplacement reactions ${ }^{24,25}$ are systematically used to design complex autonomous behaviors ${ }^{26-30}$. In a strand-displacement reaction, complementary short single-stranded regions (toeholds) colocalize separate DNA molecules to facilitate branch migration processes, which can reconfigure the molecule or release a previously sequestered single-stranded species into solution. Single-stranded products can then participate in downstream strand-displacement reactions, allowing complex cascaded circuits to be constructed, including digital circuits that evaluate boolean $\operatorname{logic}^{26}$ as well as analog circuits that modulate oligonucleotide concentrations ${ }^{27,28}$. Recently, mixed analog/digital strand-displacement circuits involving $>100$ different strands have been demonstrated ${ }^{30}$. In principle, arbitrary well-mixed chemical reaction network dynamics can be systematically implemented using DNA strand-displacement circuits $^{29}$. Such DNA circuits could potentially act as control elements for other molecular processes, such as DNA selfassembly reactions.
In order to combine the structural and dynamic aspects of DNA nanotechnology, it is necessary to identify features that allow self-assembly to proceed robustly at a constant temperature and to engineer mechanisms whereby the control signals trigger specific self-assembly reactions or structural changes. For nonautonomous systems, assembly steps, disassembly steps and conformational changes within structures formed by annealing can be effected by subsequent manual addition of control strands under isothermal conditions ${ }^{13,24-33}$; assembly can also be used for read-out ${ }^{34}$. Such isothermal self-assembly reactions could, in principle, be controlled autonomously by more complex transcriptional circuitry ${ }^{35,36}$. Additionally, isothermal selfassembly of DNA nanostructures have been demonstrated using hairpin opening reactions ${ }^{28,37-39}$, and one hairpin-based selfassembly design has even been demonstrated to function inside living cells ${ }^{20}$.

Here, we integrate two molecular programming paradigmsDNA tile self-assembly $15,17-19,40-42$ and DNA stranddisplacement circuits ${ }^{25-27,29,30,43-45}$-that have each been well characterized theoretically and experimentally. Our specific goal here is to show that in an autonomous isothermal reaction, an upstream strand-displacement control circuit can release a singlestranded signal molecule that activates specific tiles in a downstream self-assembly reaction, thus allowing control over the composition and timing of a fabrication process. For the upstream circuit, we chose an entropy-driven catalyst that releases its output at a rate controlled by an input catalyst strand ${ }^{27}$, while for downstream self-assembly, we chose a doublecrossover tile that polymerizes to form a DNA nanotube structurally analogous to biological microtubules ${ }^{40}$. The integrated system demonstrates effective control over the assembly process: in the absence of the input catalyst strand, the mixture of circuit components and precursor tiles does not produce DNA nanotubes, while with the addition of even a substoichiometric amount of input, the precursor tiles are fully converted. The resulting DNA nanotubes can be $>10 \mu \mathrm{m}$ long, as visualized in real-time through total internal reflection fluorescence (TIRF) microscopy. These structures are estimated to incorporate $>4,000$ DNA monomer tiles with a total mass of $>200 \mathrm{MDa}$.

\section{Results}

DNA tile-based nanotube self-assembly. DNA double-crossover tiles (schematically shown in Fig. 1a) were the first motifs used to demonstrate large-scale DNA self-assembly ${ }^{15,46}$. The tile consists of a rigid core containing two parallel DNA duplex helices that display four short 'sticky ends' (labeled $A, A^{\star}, B$ and $B^{\star}$ in Fig. 1). The sticky ends allow multiple copies of the tile to self-assemble into periodic lattices or nanotubes. Owing to the rigidity of the tile, complementary sticky ends on a single tile will not interact.

Several related double-crossover tile motifs have been designed and experimentally demonstrated; these vary in the details of the crossover spacing and oligonucleotide orientations ${ }^{15,46}$. We use a particular type of tile motif known as DAE-E. In addition to its simplicity (previously shown to self-assemble into DNA nanotubes with just a single tile type ${ }^{18,40}$ ), this motif also possesses an architecture amenable to integration with stranddisplacement circuits. The DAE-E motif determines the lengths and complementarities of the oligonucleotide domains, but the exact sequences of the core and the sticky ends can be varied according to design. Here, we used the core sequences from the 'UE' tile in the study by Rothemund et al. ${ }^{18}$, but with different sticky-end sequences.

Self-assembly is typically achieved through a thermal annealing process, in which a solution containing all requisite strands are 

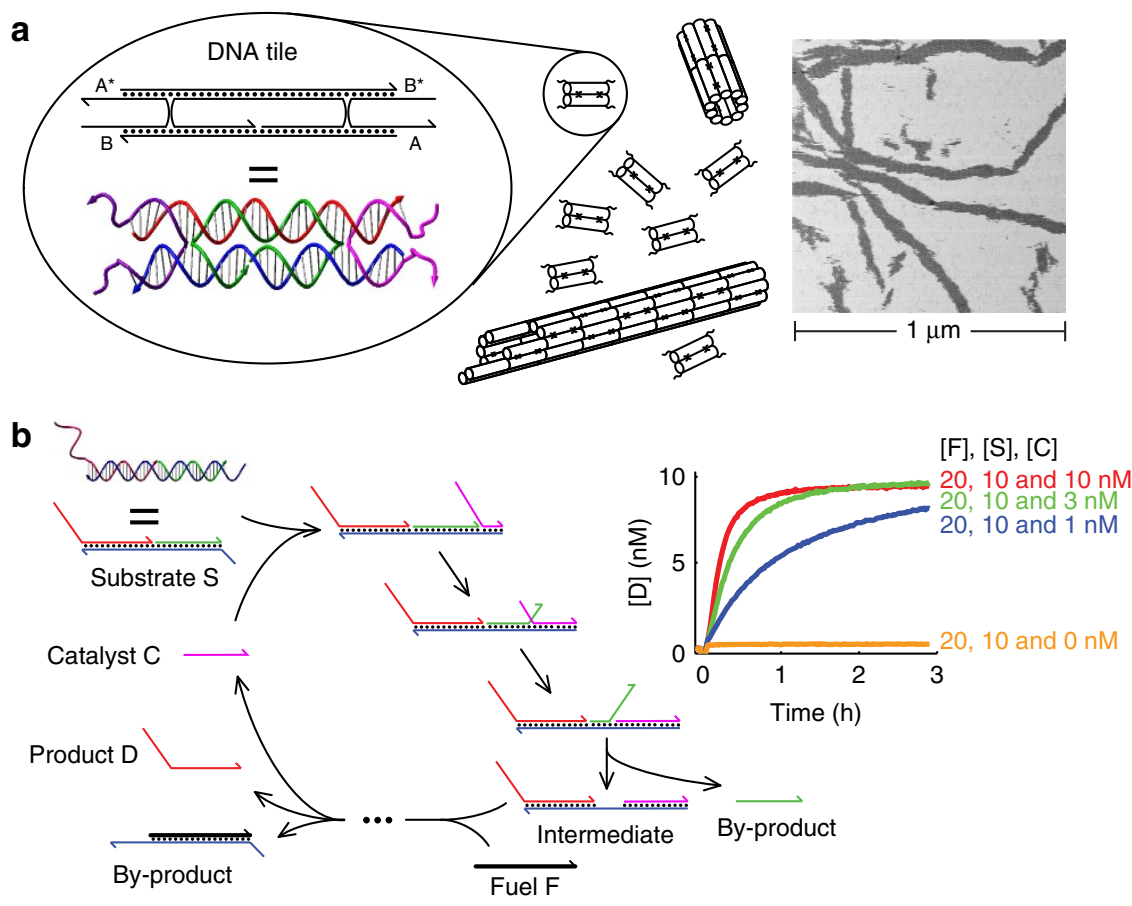

Figure 1 | Structural and dynamic DNA nanotechnology (a) Double-crossover DNA complexes (tiles) self-assemble into DNA nanotubes. Each tile possesses four single-stranded regions, known as 'sticky ends', each 5 nt long. Here, they are labeled $A, A^{\star}, B$ and $B^{\star}$, with $A$ being complementary to $A^{\star}$ and $B$ being complementary to $B^{\star}$. Owing to the intrinsic curvature at the positions where different tiles join, the tiles self-assemble into a one-dimensional DNA nanotube ${ }^{18,40}$. The nanotubes are typically observed to be between five to seven tiles wide (five-tile wide nanotubes are shown). Inset shows an atomic force microscopy (AFM) image of DNA nanotubes formed through a thermal annealing process; tubes break open upon adsorption to the mica surface. (b) A DNA catalyst expedites the release of multiple identical product oligonucleotides from a multi-stranded reactant complex. Inset shows a $25^{\circ} \mathrm{C}$ fluorescence assay of the kinetics of product release at three different substrate-to-catalyst ratios, using a fluorescent reporter that stoichiometrically reacts with the product $D$ (see Supplementary Fig. S3 for more details).

heated to $\sim 95^{\circ} \mathrm{C}$, then cooled slowly (over the course of $\sim 1.5 \mathrm{~h}$ ) to room temperature. The tile itself assembles at $\sim 60^{\circ} \mathrm{C}$, while the higher-order structures assemble at $\sim 40{ }^{\circ} \mathrm{C}$ (see ref. 18 and Supplementary Figs S1 and S2 for polyacrylamide gel electrophoresis and UV absorbance melting curves); this separation of formation temperature and the slow annealing processes ensures that tiles are formed before lattices, and is thought to facilitate reliable self-assembly. In contrast, mixture of strands at room temperature does not yield well-formed nanotubes, consistent with predictions of kinetic traps in similar molecules ${ }^{47}$. However, if tiles can be pre-formed and kept at room temperature, but prevented from hierarchical self-assembly, then subsequent isothermal activation of these tiles could lead to room temperature self-assembly.

DNA strand-displacement-based catalysis. Strand-displacement reactions can be systematically engineered to form biomolecular circuits that exhibit digital ${ }^{26}$ or analog ${ }^{27}$ logic. Figure $1 \mathrm{~b}$ shows the reaction cycle for a hybridization catalyst system that serves as an analog signal amplifier ${ }^{27}$. A multi-stranded DNA molecule, called the substrate $S$, and a single-stranded molecule, called the fuel $F$, are initially in a metastable mixture in which it is thermodynamically favorable for them to react, but, lacking toeholds, they do so at a negligible rate. However, the $S$ and $F$ can react with the help of another single-stranded molecule, called the catalyst $C$, which is not consumed in the reaction. $C$ first binds to $S$ and releases a by-product while simultaneously revealing a single-stranded toehold in the intermediate species. The singlestranded toehold provides an initiation site for reaction between the intermediate and $F$, and then $F$ displaces both $C$ and a single- stranded product $D$. The sequence of $D$ is independent of that of $C$. The released $C$ can subsequently react with other molecules of $S$ and engage in multiple turnover events; the concentration of $C$ is effectively amplified into a larger concentration of product $D$. One important feature of the catalytic system is that the sequence of $D$ is independent of that of $C$-given desired sequences for $D$ and $C, S$ and $F$ can be systematically constructed to allow $C$ to catalyze the release of $D$.

The catalytic reaction proceeds isothermally with kinetics within an order of magnitude of standard hybridization (Fig. 1b $)^{27}$. Each catalyst molecule, on average, yields the release of $>100$ product molecules (Supplementary Fig. S3), and functions robustly in the presence of other nucleic acids ${ }^{48}$. There is a slight amount of uncatalyzed (leak) reaction (that is, reaction between $F$ and $S$ in absence of $C$, as characterized by the accumulation of $D$ ); at the $10 \mathrm{nM}$ concentrations, the half-life of the leak reaction is expected to be $\sim 2$ months ${ }^{27}$.

In solution, long single-stranded regions (such as $F$ and part of $D$ in the catalyst system) could potentially interact due to spurious sequence complementarity. Consequently, careful sequence design is necessary in order to ensure that few unintended hybridization reactions occur (Table 1). Software such as NUPACK or DD ${ }^{49,50}$ can be used to design sequences and/or verify the lack of interaction between orthogonal sequences.

Integration challenges. Our approach to isothermal self-assembly relies on integrating tile-based assembly and DNA strand-displacement circuits: the product of the DNA catalyst circuit triggers the formation of active double-crossover tiles competent for 


\begin{tabular}{|c|c|c|}
\hline Domain & Sequence & Length (nt) \\
\hline 1 & $5^{\prime}-$ ATAGATCC- $3^{\prime}$ & 8 \\
\hline 2 & $5^{\prime}-\mathrm{TGATAGC}-3^{\prime}$ & 7 \\
\hline 3 & $5^{\prime}-\mathrm{GAGACCT}-3^{\prime}$ & 7 \\
\hline 4 & $5^{\prime}-\mathrm{AGCAACC}-3^{\prime}$ & 7 \\
\hline 5 & $5^{\prime}-$ TGAAACCA $-3^{\prime}$ & 8 \\
\hline 6 & $5^{\prime}-$ CGTTAAGG- $3^{\prime}$ & 8 \\
\hline 7 & $5^{\prime}-\mathrm{ACGACGCAATTCT}-3^{\prime}$ & 13 \\
\hline 8 & $5^{\prime}-\mathrm{CACATCGG}-3^{\prime}$ & 8 \\
\hline 9 & $5^{\prime}-\mathrm{ACGAGTAG}-3^{\prime}$ & 8 \\
\hline$A$ & $5^{\prime}-\mathrm{ATACC}-3^{\prime}$ & 5 \\
\hline B & $5^{\prime}-\mathrm{GAATT}-3^{\prime}$ & 5 \\
\hline 11 & $5^{\prime}-\mathrm{CCCTC}-3^{\prime}$ & 5 \\
\hline 12 & $5^{\prime}-\mathrm{ATACAATACCC}-3^{\prime}$ & 11 \\
\hline 13 & $5^{\prime}-$ TCACCATG $-3^{\prime}$ & 8 \\
\hline
\end{tabular}

self-assembly from inactive precursor tiles. We consider three methods for triggering inactive precursor tiles. Precursor tiles could be unable to self-assemble because (1) they lack a component necessary for self-assembly, or because (2) they contain an extra protecting group that prevents self-assembly. In the former case, the DNA circuit must provide the lacking component, and in the latter, the DNA circuit must provide a deprotector that removes the protecting group. These two approaches may also be combined by constructing a precursor tile that (3) both lacks a component and possesses a protecting group.

Tile activation design and experiments. The DAE-E doublecrossover tile is composed of five different strands; the three central ones contribute structural rigidity, while the outer two contain sticky ends that effect assembly logic. Removal of either or both of the two sticky-end-bearing strands would result in an incomplete precursor tile with no designed mechanism for selfassembly (Fig. 2a). When these sticky-end-bearing strands are subsequently introduced as activators, they hybridize to the incomplete tile to form the standard double-crossover structure (see Supplementary Note 1). This approach to isothermally triggering DNA nanotube growth is known as method 1: activation.

We verified by AFM and TIRF microscopy that incomplete tiles lacking either sticky-end-bearing strand do not self-assemble (Fig. 2c). When both activator strands are present, DNA nanotubes of $>10 \mu \mathrm{m}$ long are observed to assemble over the course of $2 \mathrm{~h}$. These results were further verified by native polyacrylamide gel electrophoresis (Supplementary Fig. S1) and additional AFM and TIRF (Supplementary Figs S4 and S5) experiments.

Tile deprotection design. The sticky ends of the double-crossover tile hybridize to the sticky ends of other tiles to enable hierarchical self-assembly. Pre-hybridization of one or more protector strands to these sticky ends should result in a protected tile that is incapable of self-assembly. Introduction of complements to displace the protecting strands reveals the sticky ends and enables the deprotected tile to self-assemble (Supplementary Fig. S6). This approach to isothermally triggering DNA nanotube growth is known as method 2: deprotection.

However, the short lengths of the sticky ends in the standard DAE-E tile $(5 \mathrm{nt})$ do not bind stably to their complements at room temperature; thus, implementation of this method would require either operation at low temperatures (for example, $4^{\circ} \mathrm{C}$ ) or revision to the tile architecture. Consequently, we chose not to experimentally pursue this approach here.
Tile deprotection and activation design and experiments. Figure $2 \mathrm{~b}$ shows the design of a precursor tile that reacts with its trigger via both activation and deprotection. The protected tile lacks one of the three central structural strands of the doublecrossover tile, so that each of the two protectors can stably bind to their respective sticky-end targets. The missing tile strand serves as the deprotector that displaces the protectors via a process of toehold exchange ${ }^{25,27}$. This approach to isothermally triggering DNA nanotube growth is known as method 3: deprotection and activation.

In order for the deprotector to displace the two protector species, it is necessary for it to hybridize to the central $3^{*}$ domain and subsequently undergo two independent branch migration processes that end in the spontaneous dissociation of both protectors. In doing so, the deprotector strand must wind around the central tile strand twice within the tile's 21-base-pair central region between the crossovers. The kinetics of the deprotection process thus was not known a priori: on one hand, this threading process could be sterically and electrostatically hindered; on the other, the threading is a unimolecular process and could be faster than the bimolecular toehold initiation process at reasonably low concentrations (see Supplementary Note 2).

Figure $2 \mathrm{~d}$ shows that the system functions as designed: DNA nanotubes are observed only when the protected tile reacts with the deprotector. Over the course of $2 \mathrm{~h}$, DNA nanotubes over $10 \mu \mathrm{m}$ long are observed to assemble. Any kinetic slowdown of the strand-displacement process due to the threading was not observable on this time scale. Native polyacrylamide gel electrophoresis (Supplementary Fig. S1) and melt curves (Supplementary Fig. S2) further support the claim that protected tiles are functionally and structurally distinct from the deprotected tiles.

AFM images in Fig. 2d showed that there were a number of small assemblies in addition to the DNA nanotubes after the protected tile was deprotected. Possibly, these assemblies were too small to 'wrap around' and form a tube, and thus the DNA nanotube elongation could not occur. Alternatively, these small structures may form only on the surface of the mica during imaging, and are not present in solution. Yet another possibility is that these small assemblies are fragments of DNA nanotubes that broke off as the nanotubes deposited on mica.

Catalyzed tube formation using method 3. Modularity is the primary challenge in integrating the DNA strand-displacement catalysis system with either of our two methods for isothermally triggering DNA nanotube self-assembly. While it is relatively easy to independently design a catalyst system and a triggered nanotube assembly system, combining the two so that the former feeds forward into the latter requires additional consideration for potential side reactions. In the original catalyst system ${ }^{27}$, half of the product strand is single-stranded even as part of the substrate complex, while the single-stranded fuel shares the sequence of the other half of the product strand. When this product strand is also the trigger for the precursor tiles, there is a potential for the single-stranded domains of the substrate or the fuel to spuriously interact with the precursor tiles.

The sequences of the activators for method 1 necessarily include the sticky ends that enable self-assembly of the DAE-E tile, and Supplementary Fig. S7 shows designs that illustrate the difficulty of integrating method 1 and upstream circuits, due to expected remote toehold interactions ${ }^{51}$. In contrast, the sequence of the deprotector for method 3 is independent in sequence of the sticky ends. Consequently, we judged that the latter would be less likely to exhibit unwanted interactions between different components, and chose to experimentally pursue the latter (Fig. 3a). 
a
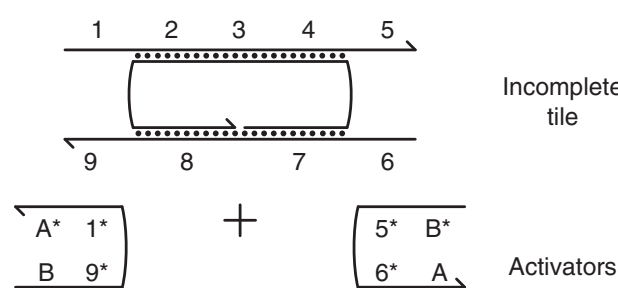

$25^{\circ} \mathrm{C} \downarrow$
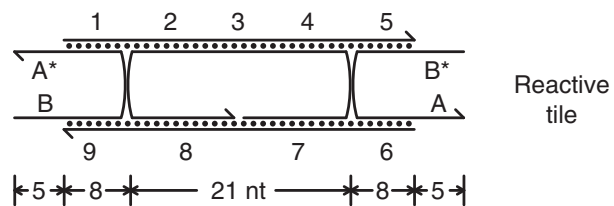
tile

tile

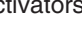

(1) b

Method 3: deprotection + activation



$25^{\circ} \mathrm{C}$

By-products

c
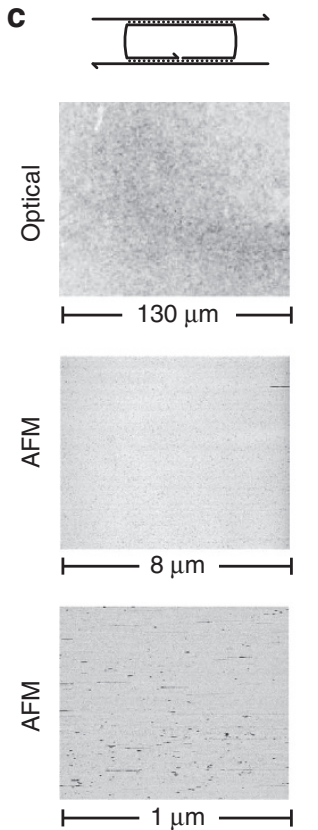


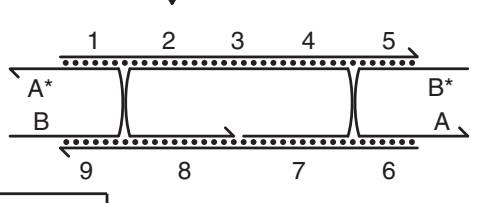

Reactive

tile
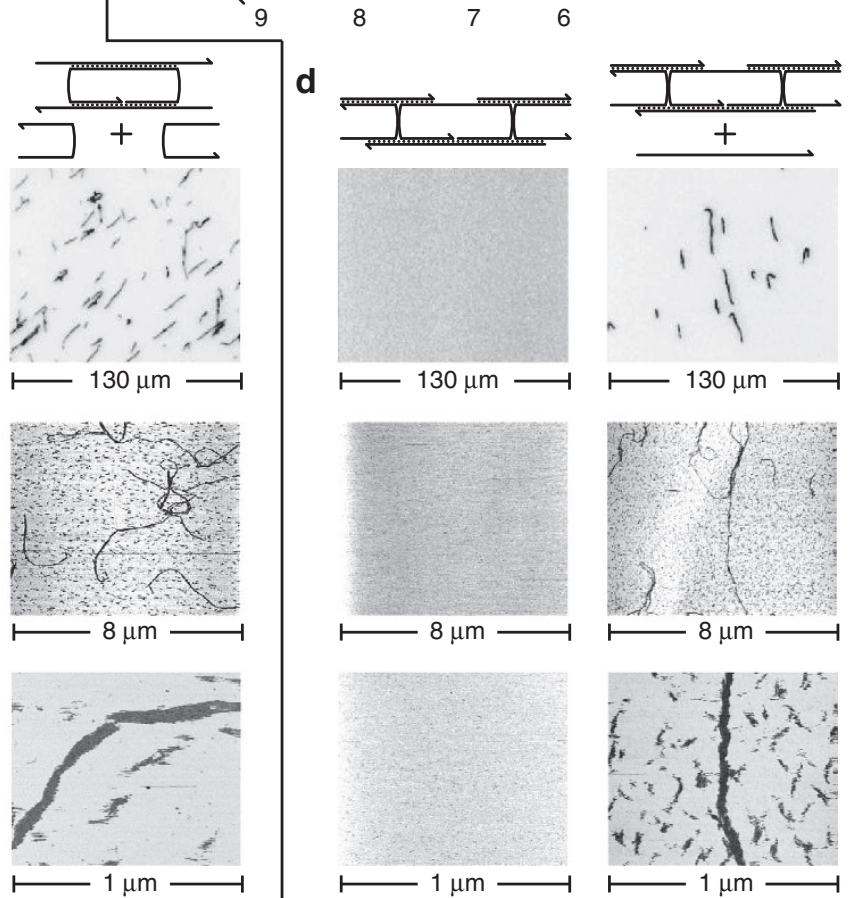

Figure 2 | Methods for isothermal triggering of DNA nanotube self-assembly. (a) Method 1: when the two sticky-end-bearing strands are absent, the three-stranded complex forms an incomplete tile that does not self-assemble. When the two missing strands (activators) are subsequently introduced, they hybridize to the incomplete tile to form the complete (reactive) tile, which then self-assembles into DNA nanotubes. (b) Method 3: an alternative method of isothermally triggering DNA self-assembly involves a protected tile in which the top two sticky ends are pre-hybridized to protector strands. Exogeneous deprotector will react with the protected tile via strand-displacement ${ }^{24,25}$ and yield the reactive tile as well as two by-product strands. (c) Experimental results on method 1 using TIRF microscopy or AFM. In all experiments, the concentrations of all species were $200 \mathrm{nM}$ and reactions were performed at room temperature $\left(25^{\circ} \mathrm{C}\right)$. For all TIRF experiments, a Cy3-modified version of the central tile strand was used. Both activators are needed for the reactive tile to form and for DNA nanotubes to self-assemble. (d) Experimental results on method 3. Addition of the deprotector causes self-assembly of DNA nanotubes.

Figure $3 \mathrm{~b}$ shows AFM microscropy results for the integrated system; fuel, substrate and protected tile do not react significantly in the absence of the catalyst, and no DNA nanotubes are observed. With a sub-stoichiometric quantity of catalyst $(20 \mathrm{nM}$, compared with $\geq 200 \mathrm{nM}$ of other reagents), DNA nanotubes in excess of $10 \mu \mathrm{m}$ in length are observed.

Previous characterization of the upstream catalyst system found that there can be a small amount of uncatalyzed reaction (leak) between the fuel and substrate that will spontaneously produce $D$ even in absence of catalyst $C$ (refs 27,48 ). To prevent this from resulting in spontaneous assembly of DNA nanotubes, we constructed a 'sink' complex that acts as a competitive threshold to absorb a stoichiometric quantity of deprotector (Fig. 3c). In the experiments reported here, the sink may not have been necessary, both because (1) the leak of the catalyst reaction was low, (2) a low concentration of active tiles resulting from residual leak would be kinetically hindered from self-assembly due to the nucleation barrier (c.f. ref. 22, Supplementary Figs S5, S8, and S9), and (3) our modeling, described in the next section, suggests that absorption by the sink may not have been faster than deprotection, to our surprise.

To directly observe the dynamics of triggered and catalyzed self-assembly, we took movies of several samples using optical (TIRF) microscopy (Fig. 4 and Supplementary Movies 1-3). 
a
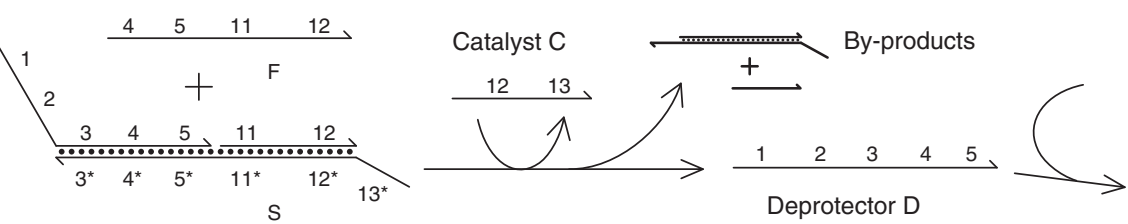

Protected tile

b

No catalyst (No sink)


Reactive tile

$+$

By-products

C

Sink

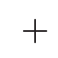

By-products

Figure 3 | Upstream control circuit. (a) A non-covalent catalytic system that produces the deprotector for method 3 as a product, based on the study by Zhang et al. ${ }^{27}$ The catalyst, an oligonucleotide with sequence independent of the deprotector, speeds up the strand-displacement reaction between reactants $F$ and $S$ to release of the deprotector. As part of the complex, the deprotector is mostly double-stranded and will not react with the protected tile. (b) AFM results on the integrated reaction network. The left panel shows the network in absence of catalyst (2-day reaction time), and the right panel shows the network in the presence of $20 \mathrm{nM}$ catalyst ( $2 \mathrm{~h}$ reaction time). In both panels, the initial concentration of the protected tile was $200 \mathrm{nM}$, of $F$ was $440 \mathrm{nM}$, of $S$ was $220 \mathrm{nM}$. The concentration of the catalyst in the right panel was $20 \mathrm{nM}, 0.1 \times$ of the limiting reagent (protected tile). DNA nanotubes form in the presence of catalyst, and do not form in the absence of catalyst. (c) The sink complex serves as a competitive threshold for the deprotector to prevent small amounts of released deprotector from causing DNA nanotube assembly.
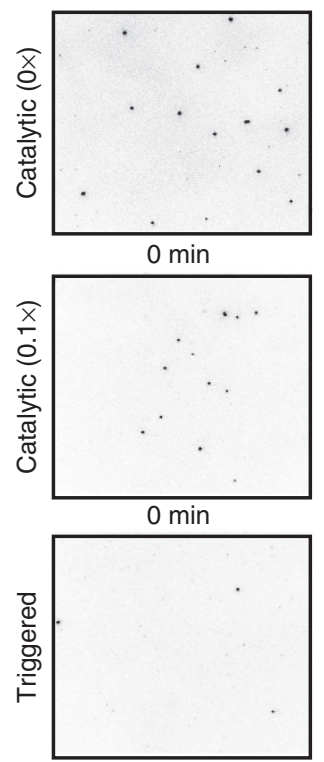

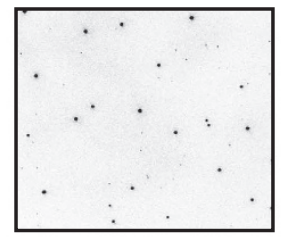

$5 \mathrm{~min}$

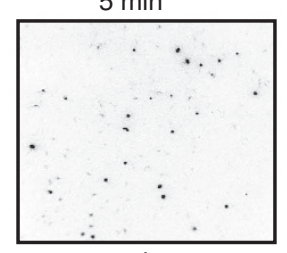

5 min

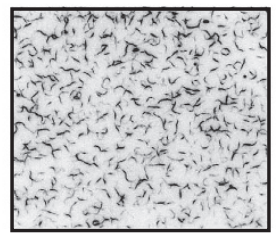

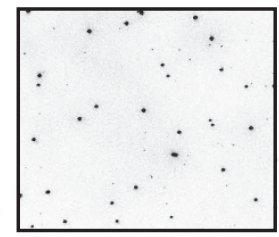

$10 \mathrm{~min}$
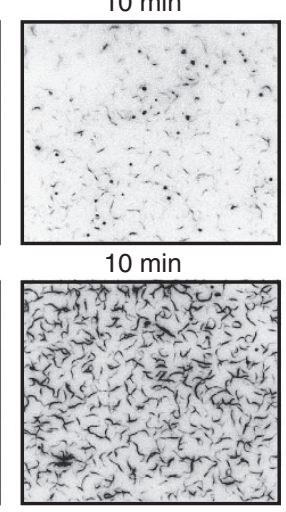

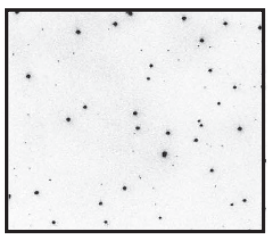

$15 \min$

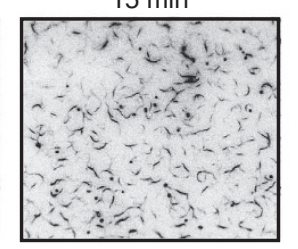

15 min

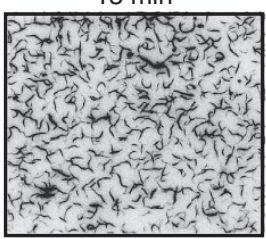

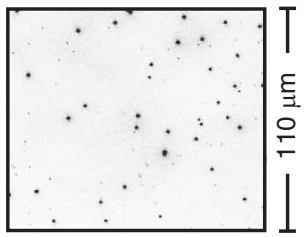

$20 \mathrm{~min}$

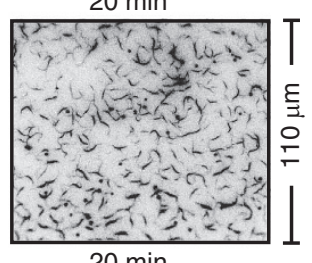

$20 \mathrm{~min}$

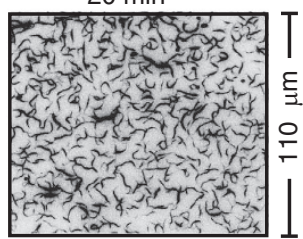

Figure 4 | Time-lapse TIRF microscopy images. These images are timepoints of TIRF movies (see Supplementary Movies 1-3). The bright circular spots correspond to fluorescent beads used for position and focus reference; the number of bright spots increase through the course of the experiments because

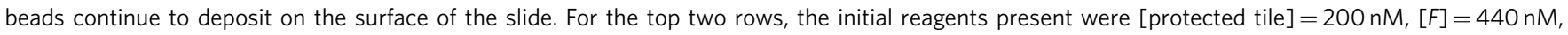
$[S]=220 \mathrm{nM}$, and $[\mathrm{sink}]=40 \mathrm{nM} ; 20 \mathrm{nM}$ catalyst was introduced at time $t \approx 0$. For the bottom row, only [protected tile] $=200 \mathrm{nM}$ was initially present; $220 \mathrm{nM}$ deprotector was introduced at time $t \approx 0$. No DNA nanotubes form within the time frame of the experiment when no catalyst was present. When a small amount $(20 \mathrm{nM})$ of catalyst was added, DNA nanotubes formed slowly, initially limited by nucleation due to low active tile concentration. From this, it can be inferred that the upstream catalyst system possesses multiple turnover, because the catalyst concentration is lower than the sink concentration. This multiple turnover is consistent with fluorimeter assay of the upstream catalyst (see Supplementary Fig. S3). When a super-stoichiometric quantity of trigger was added, DNA nanotubes formed immediately.

Samples were prepared in the presence of methylcellulose as a crowding agent to help confine DNA tubes to the surface where they can be seen by TIRF. As a consequence, tubes that nucleate and grow in solution to a large enough size will subsequently settle on the surface, often (thanks to the crowding agent) forming bundles with other deposited tubes that appear as increasing the length and fluorescence brightness of filaments during the course of the movies. When the inactive tiles and the catalytic subsystem is prepared in the absence of the catalyst strand, no tube formation is observed (Fig. 4, top, and Supplementary Movie 1). The same preparation, followed by addition of a sub-stoichiometric concentration of catalyst $(0.1 \times)$, 
results in a substantial amount of DNA tubes within 20 min (Fig. 4, middle, and Supplementary Movie 2). Movies of direct triggering of inactivated tiles (Fig. 4, bottom, and Supplementary Movie 3) indicate the fastest rate for tube nucleation, growth, and deposition under these experimental conditions, suggesting a roughly $10 \mathrm{~min}$ delay for the catalytic subsystem to produce a sufficient amount of deprotector.

To investigate the kinetics in greater detail, we performed a similar set of experiments but with six different experimental conditions (Supplementary Movie 4). From each movie, we estimated the total amount of DNA in tube form as a function of time (Fig. 5) by assuming that normalized photon counts in each movie image are linearly related to the amount of deprotected monomer incorporated into tubes (see Supplementary Methods for details).

Kinetic models of catalyzed tube formation. To determine whether the TIRF movies of the integrated catalytic DNA nanotube system are consistent with our understanding of the various sub-processes, we constructed a simplified kinetic model of the system. The reactions suggested here, and their rate constants, are intended only as a 'sanity check' for our qualitative mechanistic interpretation of the catalyzed DNA nanotube formation process, and should not be overinterpreted. The model reactions can be divided into two groups, one set for the catalysis subsystem:

$$
\begin{gathered}
S+C \underset{k_{\mathrm{bi} 2}}{\stackrel{k_{\mathrm{bi1}}}{\rightleftharpoons}} \operatorname{Int}+B 1 \\
\operatorname{Int}+F \stackrel{k_{\mathrm{bi2}}}{\longrightarrow} \operatorname{Int} 2+D \\
\operatorname{Int} 2 \underset{k_{\mathrm{bi} 1}}{\stackrel{k_{\text {uni }}}{\rightleftharpoons}} C+B 2 \\
F+S \stackrel{k_{\text {leak }}}{\longrightarrow} B 1+B 2+D
\end{gathered}
$$

and the other for the nucleation of DNA nanotubes and their subsequent elongation:

$$
\begin{gathered}
D+\operatorname{Sink} \stackrel{k_{\text {sink }}}{\rightarrow} \varnothing \\
D+\mathrm{PT} \stackrel{k_{\text {deprot }}}{\rightarrow} \text { Monomer } \\
n \cdot \text { Monomer } \stackrel{k_{\text {nuc }}}{\longrightarrow} \text { Nanotube } \\
\text { Nanotube }+ \text { Monomer } \stackrel{k_{\text {elong }}}{\longrightarrow} \text { Nanotube }
\end{gathered}
$$

Int and Int 2 correspond to two intermediates of the catalytic reaction, $B 1$ and $B 2$, respectively, refer to the single-stranded and double-stranded by-products of the catalytic cycle. PT denotes the protected tile, Monomer denotes the reactive tile, $n$ is the phenomenological nucleation stoichiometry, and [Nanotube] denotes the concentration of the DNA 'nanotubes' that have successfully nucleated.

For the integrated system, we used the best-fit catalysis rate constants, previous literature-reported rate constant of DNA nanotube elongation ${ }^{52}$, and fitted the remaining parameters to the data shown in Fig. 5 (Table 2, see Supplementary Note 4). The value of $k_{\text {elong }}$ for a related, but not identical, DNA nanotube was experimentally measured in the study of Hariadi et al..$^{52}$ to be $5.7 \times 10^{5}$ layers per $M$ per $s$; our DNA nanotubes appear to have a mean circumference of six tiles, so we estimate $k_{\text {elong }}=3.4 \times 10^{6} \mathrm{M}^{-1} \mathrm{~s}^{-1}$. We then fit $k_{\text {deprot }}, k_{\text {sink }}$ and $k_{\text {nuc }}$ for different values of $n$ to five of the data traces shown in Fig. 5 (the $0 \times$ catalyst control trace shown in cyan, being almost completely flat, was not fitted). For the best fit, $n=2.5$ (Fig. 5 inset), $\quad k_{\text {deprot }}=10^{7} \mathrm{M}^{-1} \mathrm{~s}^{-1}, \quad k_{\text {sink }}=10^{5} \mathrm{M}^{-1} \mathrm{~s}^{-1} \quad$ and $k_{\text {nuc }}=2.0 \times 10^{5} \mathrm{M}^{-1} \mathrm{~s}^{-1}$

In the course of our fitting, we constrained the values of $k_{\text {deprot }}$ and $k_{\text {sink }}$ to be between $10^{5} \mathrm{M}^{-1} \mathrm{~s}^{-1}$ and $10^{7} \mathrm{M}^{-1} \mathrm{~s}^{-1}$, consistent with our expectations for strand-displacement reactions with toeholds of this length ${ }^{25}$. The best-fit values of these two parameters were at the limits $\left(k_{\text {deprot }}=10^{7}, k_{\text {sink }}=10^{5}\right)$, with

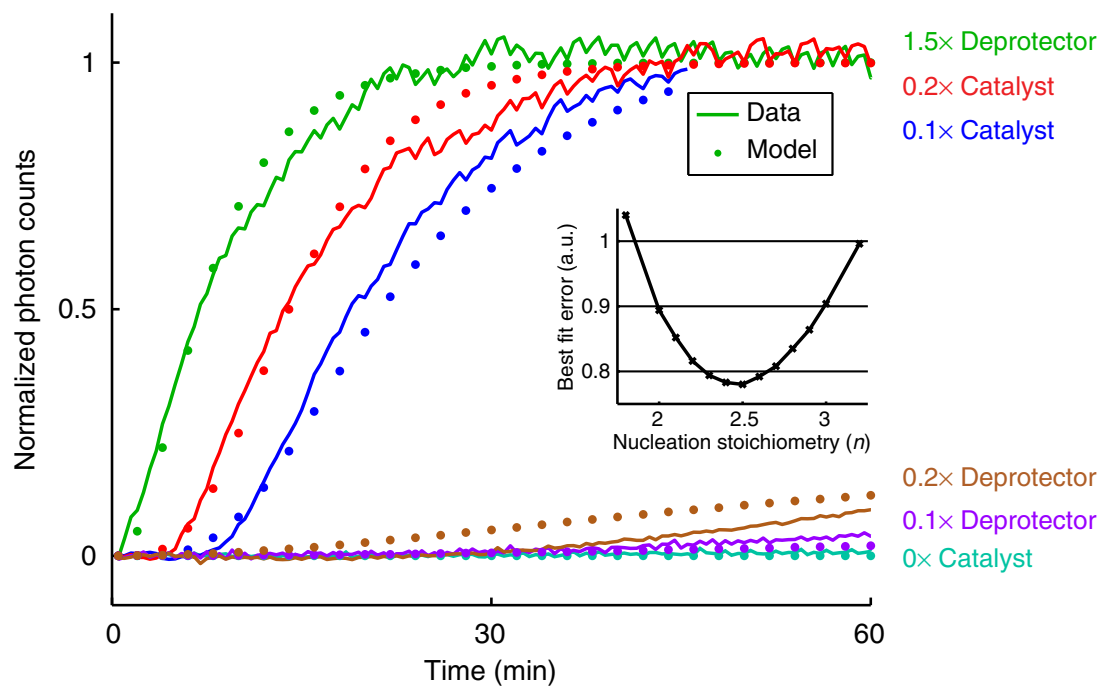

Figure 5 | Modeling and quantitation of DNA nanotube growth. Normalized photon counts of time for time-lapse TIRF microscopy images and control experiments shown as Supplementary Movie 4 (these are distinct from experiments shown in Figure 4); this value corresponds to the amount of deprotected monomers incorporated into tubes. In all experiments shown here, [protected tile] $=200 \mathrm{nM}(1 \times)$ and [sink] $=40 \mathrm{nM}(0.2 \times)$. For the green, brown, and purple traces, the listed quantity of deprotector was introduced at time $t \approx 0$. For the red and blue traces, [fuel] $=600 \mathrm{nM}(3 \times)$ and [substrate] $=300 \mathrm{nM}(1.5 \times)$ were present in solution initially, and the listed quantity of catalyst was introduced at time $t=0$. Shown in dotted lines are the expected kinetic behaviors predicted by our model using a best-fit nucleation stoichiometry value of $n=2.5$; the inset shows the relative sum of squared error for different values of $n$ (with corresponding best-fit $k_{\text {nuc }}, k_{\text {deprot }}$ and $k_{\text {sink }}$ rate constants). The model captures the qualitative differences of the different traces; quantitative agreement is limited due to many uncharacterized parameters and the simplicity/incompleteness of model reactions. 
Table 2 | Model parameters values.

\begin{tabular}{l} 
Parameter \\
\hline$k_{\text {bi1 }}$ \\
$k_{\text {bi2 }}$ \\
$k_{\text {uni }}$ \\
$k_{\text {leak }}$ \\
$k_{\text {sink }}$ \\
$k_{\text {deprot }}$ \\
$n$ \\
$k_{\text {nuc }}$ \\
$k_{\text {elong }}$
\end{tabular}

\section{Description}

$S+C$ rate constant; rxn (1) and (3); fitted

$F+$ Int rate constant; $r \times n(1)$ and (2); fitted

Int $2 \rightarrow C+B 2$ rate constant; rxn (3); fitted

$F+S$ leakage rate constant; rxn (4); fitted

$D+$ sink rate constant; rxn (5); fitted

$D+P T$ rate constant; $r \times n$ (6); fitted

Phenomenological stoichiometry of nucleation; fitted

$n \cdot$ monomer nucleation rate constant; rxn (7); fitted

Nanotube + Monomer rate constant; rxn (8); assumed
Value

$$
\begin{gathered}
5.5 \times 10^{5} \mathrm{M}^{-1} \mathrm{~s}^{-1} \\
6.2 \times 10^{6} \mathrm{M}^{-1} \mathrm{~s}^{-1} \\
4.2 \times 10^{-2} \mathrm{~s}^{-1} \\
7.4 \mathrm{M}^{-1} \mathrm{~s}^{-1} \\
10^{5} \mathrm{M}^{-1} \mathrm{~s}^{-1} \\
10^{7} \mathrm{M}^{-1} \mathrm{~s}^{-1} \\
2.5 \\
2.0 \times 10^{5} \mathrm{M}^{1-n} \mathrm{~s}^{-1} \\
3.4 \times 10^{6} \mathrm{M}^{-1} \mathrm{~s}^{-1}
\end{gathered}
$$

The parameters of the catalysis subsystem were fitted only to the catalytic data shown in Supplementary Fig. S3, which includes fluorescence reporter characterization, the data shown in Figure 1b with $10 \mathrm{nM}$ precursors, and catalytic behavior at $100 \mathrm{nM}$ precursor concentrations (see Supplementary Methods for fitting details). We initially attempted to use more simplified reaction models for the catalysis subsystem, but these models failed to qualitatively capture its kinetic behavior over the relevant range of times and concentrations. Specifically, reaction (3) is essential for capturing both the unimolecular rate-limiting nature of the catalytic reaction at high substrate concentrations, and the product inhibition (due to reverse reaction) that slows the catalysis as the reaction approaches completion. Reactions (2) and (4) are modeled as irreversible, whereas in reality some amount of reverse reaction may occur (see Supplementary Note 3). A similar model was previously explored in the study by Zhang et al. ${ }^{48}$, and rate parameters reported in that work are all within one order of magnitude of corresponding rate constants here (with differences likely attributed to differences in DNA sequence).

$k_{\text {deprot }}$ significantly higher than $k_{\text {sink }}$. This seems to indicate that the sink is not effective in reducing the leak reaction. However, the model is sensitive to changes in nucleation stoichiometry: slightly higher or lower concentrations of certain species can result in significantly different parameter values.

The model captures the qualitative features of the different traces, including (1) the characteristic shapes of the $1.5 \times$ deprotector, $0.2 \times$ catalyst and $0.1 \times$ catalyst traces (shown in green, red and blue, respectively) that are affected by the nucleation barrier and the slowdown of the catalytic system, (2) the separation of these three traces corresponding to the delayed release of deprotector molecules, and (3) the eventual rise of the $0.2 \times$ deprotector trace (brown), due to both catalytic leakage and the competition between the protected tile and the sink. We hypothesize that the quantitative fitting could be improved via (1) more detailed modeling of the tube nucleation and elongation processes, (2) modeling of surface adsorption kinetics and (3) improved characterization of the catalytic system for this particular set of sequences. Based on the time-lapse TIRF results and our model predictions, we conclude that our experimental data is consistent with and supports our rationally designed mechanism for the kinetic control of isothermal DNA nanotube assembly using a modular upstream catalyst system. In particular, multiple turnover of the catalyst is unambiguously shown by the $0.1 \times$ catalyst trace (blue) that yields significantly higher photon counts than the $0.2 \times$ deprotector trace (brown).

\section{Discussion}

By designing inert precursor double-crossover tiles that are activated through isothermal strand-displacement processes, and by using an upstream strand-displacement circuit to control the timing of DNA nanotube self-assembly, we have demonstrated room-temperature self-assembly of DNA nanotubes over $10 \mu \mathrm{m}$ long from nanometer-scale components. It is reasonable to believe that the methods and results here may be robust to a variety of temperatures and environmental conditions, because we did not fine-tune any part of the design process for optimal function at room temperature; previous DNA strand-displacement systems exhibit temperature and salinity robustness ${ }^{27,48,53}$. More fundamentally, we have established a link between the well-studied molecular programming paradigms of stranddisplacement circuits and tile-based self-assembly. As the former paradigm enables complex dynamical behavior and the latter enables complex spatial structure formation, their integration has the potential to enable a rich class of isothermal molecular systems with complex spatiotemporal behaviors.
To go beyond the particular example demonstrated here and to establish the generality of our approach, a systematic method of coupling DNA tile systems and DNA strand-displacement circuits must be developed. This could be challenging because, on the one hand, there are many distinct DNA tile motifs (for example, several variants of double-crossover molecules ${ }^{15,46}$, multi-crossover molecules ${ }^{17,54}$, rigid-junction tiles ${ }^{55}$, singlestranded tiles ${ }^{56,57}$ ) and RNA tiles ${ }^{58}$. On the other hand, systematic methods to compile abstract biochemical circuit specifications into DNA strand-displacement systems employ a variety of standardized domain architectures to avoid unintentional interactions between dynamic species $29,43,45,59$, and it will be necessary to verify that undesired interactions can be systematically avoided when arbitrary tile sets are interfaced to arbitrary circuits. Although these challenges do not appear insurmountable, especially with the aid of automated software tools $^{43}$, they will require care.

A theoretical framework is needed to characterize what molecular behaviors can or cannot be achieved by integrated DNA tile and strand-displacement systems. The foundations of such a framework already exist in the extensive theoretical characterization of both tile systems (for example, arbitrary computable shapes can be self-assembled using small tile sets ${ }^{41,42}$ ) and strand-displacement systems (for example, arbitrary formal chemical reaction network dynamics can be implemented but suffer certain logical limitations ${ }^{29,60}$ ). Nonetheless, there is considerable flexibility to consider distinct mechanisms for coupling tile systems and strand-displacement systems, and these choices may have significant implications for the theoretical capabilities of the class of molecular programs obtained. For example, here we showed that signals from a strand-displacement system to a tile system can control the timing of tile activation. It is not hard to imagine that a feedback signal from the tile system - such as a 'by-product' of method 3could be used to trigger further strand-displacement circuit events. A feedback signal that is released only when a tile is successfully assembled into a growing structure (rather than just when it is activated) would be even more powerful, though more difficult to implement. By further allowing strand-displacement signals to trigger and report disassembly events as well as assembly events, remarkably efficient Turing-universal computation is theoretically possible, even using just onedimensional structures ${ }^{59}$.

Beyond allowing for a theoretical characterization, systematic architectures and formal models maximally leverage experimental advances, in that lessons learned from specific experimental 
implementations can be applied to a wide range of potential systems whose behaviors can be formally characterized. For example, DNA tile self-assembly was proposed as an abstract model of computation and shown to be Turing-universal for both computation and for construction ${ }^{41,42}$, guiding experimental demonstrations to rapidly increasing complexity $15,18,19$. Analogously, DNA strand-displacement circuits have been formalized as a programming language and shown to be capable of implementing arbitrary chemical reaction network dynamics, including arbitrary digital and analog circuits ${ }^{29,43}$, facilitating the experimental advance from tens of strands to hundreds ${ }^{26,30}$. Key to both work is that generic molecular components can be conceptualized as motifs whose behavior is largely independent of specific (well-designed) sequence choices-conceptual design can be performed at the domain level, then implemented systematically. These properties make it meaningful to discuss full-fledged programming languages and compilers ${ }^{43,50}$ for molecular programming architectures.

Molecular programming paradigms that combine solutionphase circuitry with structural self-assembly are not limited to the combination of tile systems and strand-displacement cascades. Indeed, interacting hairpin systems ${ }^{28,37-39}$ naturally and seamlessly integrate circuitry, assembly, and disassembly reactions, and have been experimentally demonstrated for tasks such as triggered and catalytic assembly, signal amplification circuitry and molecular motors. However, theoretical characterization of what can and cannot be achieved using interacting hairpin systems lags behind the experimental demonstrations. Thus, an important target for future research will be to develop theory, software and experimental practice that integrates all three molecular programming paradigms. Such advances will facilitate the application of molecular programming technologies to real-world problems ${ }^{38,44}$.

\section{Methods}

DNA oligonucleotides. DNA oligonucleotides used in this study were purchased from Integrated DNA Technologies (IDT), and HPLC purified by IDT. Where applicable, fluorophores were attached by IDT as well.

Buffer conditions. Individual DNA oligonucleotides were resuspended and stored in TE buffer ( $10 \mathrm{mM}$ Tris-HCl pH balanced to 8.0 , with $1 \mathrm{mM} \mathrm{EDTA} \cdot \mathrm{Na}_{2}$, purchased as $100 \times$ stock from Sigma-Aldrich) at $4{ }^{\circ} \mathrm{C}$. Directly preceding experiments, TE buffer with $62.5 \mathrm{mM} \mathrm{MgCl}_{2}$ was added at $1: 4$ ratio to the sample, achieving a final $\mathrm{MgCl}_{2}$ concentration of $12.5 \mathrm{mM}$. As roughly $1 \mathrm{mM}$ of the $\mathrm{Mg}^{2}+$ is chelated by the EDTA present in solution, the free concentration of $\mathrm{Mg}^{2+}$ is estimated to be $11.5 \mathrm{mM}$. All experiments and purifications were performed at $25^{\circ} \mathrm{C}$.

Complex purifications. Multi-stranded complexes (namely the incomplete tile, the protected tile, the catalytic substrate, and the trigger sink) were purified inhouse by non-denaturing PAGE as follows: oligonucleotides needed for each sample were prepared with nominally correct stoichiometry at $20 \mu \mathrm{M}$ and annealed with an Eppendorf Mastercycler Gradient thermocycler. The solutions were brought down from $95^{\circ} \mathrm{C}$ to $20^{\circ} \mathrm{C}$ at a constant rate over the course of $75 \mathrm{~min}$. The solution were then run on $12 \%$ non-denaturing PAGE at $180 \mathrm{~V}$ for $6 \mathrm{~h}$. The proper bands were cut out and eluted in $1 \mathrm{ml} \mathrm{TE} / \mathrm{Mg}^{2+}$ buffer for 2 days. Typical yields ranged from 40 to $60 \%$. The concentrations of purified complexes were estimated by measuring absorbance at $260 \mathrm{~nm}$ using an Eppendorf Biophotometer, and dividing by extinction coefficients for single- and double-stranded DNA predicted by nearest-neighbor models.

Atomic force microscopy. AFM images were acquired using tapping mode on a Nanoscope IIIa (Veeco Instruments) under $1 \times \mathrm{TAE} / \mathrm{Mg}^{2+}(\mathrm{TAE}=40 \mathrm{mM}$ Tris base, $20 \mathrm{mM}$ acetic acid, and $1 \mathrm{mM}$ EDTA, pH 8.0 with $12.5 \mathrm{mM}$ Mg-acetate$4 \mathrm{H}_{2} \mathrm{O}$ ) buffer equipped with nanoAnalytics Q-control III (Asylum Research) and $110 \mu \mathrm{m}, 0.38 \mathrm{~N} \mathrm{~m}^{-1}$ spring constant SNL silicon nitride cantilever (Veeco Instruments)

Following (potential) self-assembly in the centrifuge tubes, the samples were diluted by a factor of two with $1 \times \mathrm{TAE} / \mathrm{Mg}^{2+}$. Five microlitre of this diluted sample was deposited on a freshly cleaved piece of V1 grade mica (Ted Pella),

$\sim 1 \mathrm{~cm} \times 1 \mathrm{~cm}$ in size, affixed to a 15 -mm diameter magnetic stainless steel puck
(Ted Pella) using a hot glue gun. The presence of $\mathrm{Mg}^{2+}$ ions and other multivalent cations in the buffer facilitates the formation of salt bridges between the DNA species and negatively charged mica surface. Once the DNA nanotubes are immobilized on the mica, no further reactions (assembly, end-to-end joining, disassembly, and so on) are expected to occur. An additional $30 \mu \mathrm{l}$ of $1 \times \mathrm{TAE} /$ $\mathrm{Mg}^{2+}$ was added to both sample and cantilever holder before imaging.

We imaged each sample at three or more random locations, each at least $75 \mu \mathrm{m}$ apart. At each scan location, we imaged at the 8,2 and $1 \mu \mathrm{m}$ scales. We used custom-written Matlab and Mathematica code to flatten the images by subtracting a linear fit from each scan line and matching intensity histograms between scan lines.

Total internal reflection fluorescence optical microscopy. We used fluorescence microscopy with a $130 \times 130 \mu \mathrm{m}$ field of view to visualize self-assembly reactions in real-time using a custom-built prism-based TIRF microscope equipped with autofocus and temperature controller, as previously described ${ }^{52}$

For fluorescence microscopy imaging only, the tile's central strand (with sequence $7^{\star} 4^{\star} 3^{\star} 2^{\star} 8^{\star}$ ) was $5^{\prime}$-end-labeled with a Cy3 fluorophore. The samples were excited by a 532-nm solid-state laser (CrystaLaser) weakly focused on the surface of a suprasil prism (CVI Melles Griot) upon which the microscope slide was mounted. The emitted photons were collected by a $60 \times, 1.2 \mathrm{NA}$ water immersion objective (Nikon), $20 \mathrm{~cm}$ lens tube (CVI Melles Griot) and a C9100-02 electron multiplier CCD camera (Hamamatsu). The images were analyzed using the ImageJ software (NIH) and Mathematica.

For static imaging (for example, Fig. $2 c, d$ ), a 5 - $\mu$ l drop of typically 10 -fold diluted sample was deposited between a clean microscope slide and a coverslip. The dilution factor of the static fluorescence assay is higher than that of the AFM imaging to facilitate visualization of individual nanotubes. Under $1 \times \mathrm{TAE} / \mathrm{Mg}^{2}+$ buffer, DNA nanotubes were immobilized on negatively charged clean glass surfaces.

For fluorescence movies, snapshots of which appear in Fig. 4, we flowed $6 \mu \mathrm{l}$ of the sample into a flow chamber made from a glass capillary tube (Vitrotubes) and subsequently sealed both ends of the chamber with Vaseline. DNA nanotubes were observed to interact minimally with the capillary tube; therefore, no glass passivation protocol was necessary for real-time observation of self-assembly. Owing to the brightness and slow photobleaching rate of $\mathrm{Cy} 3$, the sample reaction does not contain an oxygen scavenger mix. To guide the autofocus process, $100 \mathrm{~nm}$ fluorescent beads (Polyscience, Inc) were added at roughly $1 \mathrm{pM}$. To confine the DNA nanotubes within the evanescence field near the surface, a crowding agent $(0.3 \% w / v$ of methylcellulose, Sigma-Aldrich) was added to the solution mix. In the presence of crowding agents, the entropy of the system is maximized when all long structures are close to another surface, such as the capillary tube walls.

A side effect of this confinement strategy is that the same entropic force also favors confining DNA nanotubes to any surface, such as the surfaces of other DNA nanotubes. Consequently, at high concentrations, DNA nanotubes are observed to exhibit side-to-side joining and lateral aggregation. The increasing intensity of tubes corresponds to the lateral 'bundling' of multiple DNA nanotubes.

\section{References}

1. Mann, S. Life as a nanoscale phenomenon. Angew. Chem. Int. Ed. 47, 5306-5320 (2008).

2. Leiman, P. G., Kanamaru, S., Mesyanzhinov, V. V., Arsaka, F. \& Rossman, M. G. Structure and morphogenesis of bacteriophage T4. Cell. Mol. Life Sci. 60, 2356-2370 (2003).

3. Aldridge, P. \& Hughes, K. T. Regulation of flagellar assembly. Curr. Opin. Microbiol. 5, 160-165 (2002).

4. Desai, A. \& Mitchison, T. J. Microtubule polymerization dynamics. Annu. Rev Cell Dev. Biol. 13, 83-117 (1997).

5. Lloyd, C. \& Chan, J. Microtubules and the shape of plants to come. Nat. Rev. Mol. Cell Biol. 5, 13-23 (2004).

6. Li, R. \& Gundersen, G. G. Beyond polymer polarity: how the cytoskeleton builds a polarized cell. Nat. Rev. Mol. Cell Biol. 9, 860-873 (2008).

7. Mattila, P. K. \& Lappalainen, P. Filopodia: molecular architecture and cellular functions. Nat. Rev. Mol. Cell Biol. 9, 446-454 (2008).

8. Drexler, K. E. Molecular engineering: an approach to the development of general capabilities for molecular manipulation. Proc. Natl Acad. Sci. USA 78, 5275-5278 (1981).

9. Szostak, J. W., Bartel, D. P. \& Luisi, P. L. Synthesizing life. Nature 409, 387-390 (2001).

10. Noireaux, V. \& Libchaber, A. A vesicle bioreactor as a step toward an artificial cell assembly. Proc. Natl Acad. Sci. 101, 17669-17674 (2004).

11. Goel, A. \& Vogel, V. Harnessing biological motors to engineer systems for nanoscale transport and assembly. Nat. Nanotech. 3, 465-475 (2008).

12. Lund, K. et al. Molecular robots guided by prescriptive landscapes. Nature 465, 206-210 (2010)

13. Gu, H., Chao, J., Xiao, S. J. \& Seeman, N. C. A proximity-based programmable DNA nanoscale assembly line. Nature 465, 202-205 (2010).

14. Seeman, N. C. Structural DNA nanotechnology: growing along with NanoLetters. Nano Lett. 10, 1971-1978 (2010). 
15. Winfree, E., Liu, F., Wenzler, L. A. \& Seeman, N. C. Design and self-assembly of two-dimensional DNA crystals. Nature 394, 539-544 (1998).

16. Zheng, J. et al. From molecular to macroscopic via the rational design of a selfassembled 3D DNA crystal. Nature 461, 74-77 (2009).

17. Mao, C., LaBean, T. H., Reif, J. H. \& Seeman, N. C. Logical computation using algorithmic self-assembly of DNA triple-crossover molecules. Nature 407, 493-496 (2000).

18. Rothemund, P. W. K., Papadakis, N. \& Winfree, E. Algorithmic self-assembly of DNA Sierpinski triangles. PLoS Biol. 2, e424 (2004).

19. Barish, R. D., Schulman, R., Rothemund, P. W. K. \& Winfree, E. An information-bearing seed for nucleating algorithmic self-assembly. Proc. Natl Acad. Sci. USA 106, 6054-6059 (2009).

20. Delebecque, C. J., Lindner, A. B., Silver, P. A. \& Aldaye, F. A. Organization of intracellular reactions with rationally designed RNA assemblies. Science 333, 470-474 (2011).

21. Schulman, R., Yurke, B. \& Winfree, E. Robust self-replication of combinatorial information via crystal growth and scission. Proc. Natl Acad. Sci. 109, 6405-6410 (2012).

22. Schulman, R. \& Winfree, E. Synthesis of crystals with a programmable kinetic barrier to nucleation. Proc. Natl Acad. Sci. 104, 15236-15241 (2007).

23. Zhang, D. Y. \& Seelig, G. Dynamic DNA nanotechnology using stranddisplacement reactions. Nat. Chem. 3, 103-113 (2011).

24. Yurke, B., Turberfield, A. J., Mills, A. P., Simmel, F. C. \& Neumann, J. L. A DNA-fuelled molecular machine made of DNA. Nature 406, 605-608 (2000).

25. Zhang, D. Y. \& Winfree, E. Control of DNA strand displacement kinetics using toehold exchange. J. Am. Chem. Soc. 131, 17303-17314 (2009).

26. Seelig, G., Soloveichik, D., Zhang, D. Y. \& Winfree, E. Enzyme-free nucleic acid logic circuits. Science 314, 1585-1588 (2006).

27. Zhang, D. Y., Turberfield, A. J., Yurke, B. \& Winfree, E. Engineering entropydriven reactions and networks catalyzed by DNA. Science 318, 1121-1125 (2007).

28. Yin, P., Choi, H. M. T., Calvert, C. R. \& Pierce, N. A. Programming biomolecular self-assembly pathways. Nature 451, 318-322 (2008).

29. Soloveichik, D., Seelig, G. \& Winfree, E. DNA as a universal substrate for chemical kinetics. Proc. Natl Acad. Sci. 107, 5393-5398 (2010).

30. Qian, L., Winfree, E. \& Bruck, J. Neural network computation with DNA strand displacement cascades. Nature 475, 368-372 (2011).

31. Shin, J. S. \& Pierce, N. A. A synthetic DNA walker for molecular transport. J. Am. Chem. Soc. 126, 10834-10835 (2004).

32. Feng, L. P., Park, S. H., Reif, J. H. \& Yan, H. A two-state DNA lattice switched by DNA nanoactuator. Angew. Chem. Int. Ed. 42, 4342-4346 (2003).

33. Andersen, E. S. et al. Self-assembly of a nanoscale DNA box with a controllable lid. Nature 459, 73-76 (2009).

34. Kolpashchikov, D. M., Gerasimova, Y. V. \& Khan, M. S. DNA nanotechnology for nucleic acid analysis: DX motif-based sensor. Chembiochem 12, 2564-2567 (2011).

35. Dittmer, W. U. \& Simmel, F. C. Transcriptional control of DNA-based nanomachines. Nano Lett. 4, 689-691 (2004).

36. Kim, J. \& Winfree, E. Synthetic in vitro transcriptional oscillators. Mol. Syst. Biol. 7, 465 (2011).

37. Dirks, R. M. \& Pierce, N. A. Triggered amplification by hybridization chain reaction. Proc. Natl Acad. Sci. USA 101, 15275-1278 (2004).

38. Choi, H. M. T., Chang, J. Y., Trinh, L. A., Padilla, J. E., Fraser, S. E. \& Pierce, N. A. Programmable in situ amplification for multiplexed imaging of mRNA expression. Nat. Biotechnol. 28, 1208-1212 (2010).

39. Muscat, R. A., Bath, J. \& Turberfield, A. J. A programmable molecular robot. Nano. Lett. 11, 982-987 (2011).

40. Rothemund, P. W. K., Ekani-Nkodo, A., Papadakis, N., Fygenson, D. K. \& Winfree, E. Design and characterization of programmable DNA nanotubes. J. Am. Chem. Soc. 126, 16344-16352 (2004).

41. Rothemund, P. W. K. \& Winfree, E. The program-size complexity of selfassembled squares. Proc. 32nd Ann. ACM Symposium on Theory Comp. 459-468 (2000).

42. Soloveichik, D. \& Winfree, E. Complexity of self-assembled shapes. SIAM J. Comp. 36, 1544-1569 (2007).

43. Phillips, A. \& Cardelli, L. A programming language for composable DNA circuits. J. R. Soc. Interface 6, S419 (2009).

44. Li, B., Ellington, A. D. \& Chen, X. Rational, modular adaptation of enzyme-free DNA circuits to multiple detection methods. Nucleic. Acids Res. 39, e110 (2011).
45. Cardelli, L. Two-domain DNA strand displacement. Math. Struct. Comp. Sci. 23, 247-271 (2013).

46. Fu, T. J. \& Seeman, N. C. DNA double-crossover molecules. Biochemistry 32, 3211-3220 (1993).

47. Kumara, T. M., Nykypanchuk, D. \& Sherman, W. B. Assembly pathway analysis of DNA nanostructures and the construction of parallel motifs. Nano Lett. 8, 1971-1977 (2008).

48. Zhang, D. Y. \& Winfree, E. Robustness and modularity properties of a non-covalent DNA catalytic reaction. Nucleic. Acids Res. 38, 4182-4197 (2010).

49. Zhang, D. Y. Towards domain-based sequence design for DNA strand displacement reactions. Lect. Notes Comp. Sci. 6518, 162-175 (2011).

50. Zadeh, J. N. et al. NUPACK: analysis and design of nucleic acid systems. J. Comput. Chem. 32, 170-173 (2011).

51. Genot, A. J., Zhang, D. Y., Bath, J. \& Turberfield, A. J. Remote toehold: a mechanism for flexible control of DNA hybridization kinetics. J. Am. Chem. Soc. 133, 2177-2182 (2011).

52. Hariadi, R. F. Non-equilibrium Dynamics of DNA Nanotubes, PhD. Thesis (California Institute of Technology, 2011).

53. Zhang, D. Y., Chen, S. X. \& Yin, P. Optimizing the specificity of nucleic acid hybridization. Nat. Chem. 4, 208-214 (2012).

54. Ke, Y., Liu, Y., Zhang, J. \& Yan, H. A study of DNA tube formation mechanisms using 4-, 8-, and 12-helix DNA nanostructures. J. Am. Chem. Soc 128, 4414-4421 (2006)

55. He, Y., Chen, Y., Liu, H., Ribbe, A. E. \& Mao, C. Self-assembly of hexagonal DNA two-dimensional (2D) arrays. J. Am. Chem. Soc. 127, 12202-12203 (2005).

56. Yin, P. et al. Programming DNA tube circumferences. Science 321, 824-826 (2008).

57. Wei, B., Dai, M. \& Yin, P. Complex shapes self-assembled from single-stranded DNA tiles. Nature 485, 623-626 (2012).

58. Chworos, A. et al. Building programmable jigsaw puzzles with RNA. Science 306, 2068-2072 (2004)

59. Qian, L., Soloveichik, D. \& Winfree, E. Efficient Turing-universal computation with DNA polymers. Lect. Notes Comp. Sci. 6518, 123-140 (2011).

60. Condon, A., Hu, A. J., Manuch, J. \& Thachuk, C. Less haste, less waste: on recycling and its limits in strand displacement systems. Interface Focus 2, 512-521 (2012).

\section{Acknowledgements}

This work was supported in part by National Science Foundations grants 0832824 , 0829805, and 0728703 to E.W. This work was supported in part by a Hertz Foundation graduate fellowship, a Howard Hughes Medical Institute postdoctoral fellowship as part of the Life Sciences Research Foundation program, and NIH Award 1K99EB015331 to D.Y.Z.

\section{Author contributions}

D.Y.Z., R.F.H. and H.M.T.C. conceived and designed the experiments; D.Y.Z. and R.F.H performed the experiments and analyzed the data; D.Y.Z. and E.W. wrote the paper; E.W. guided the research.

\section{Additional information}

Supplementary Information accompanies this paper at http://www.nature.com/ naturecommunications

Competing financial interests: The authors declare no competing financial interests.

Reprints and permission information is available online at http://npg.nature.com/ reprintsandpermissions/

How to cite this article: Zhang, D. Y. et al. Integrating DNA strand-displacement circuitry with DNA tile self-assembly. Nat. Commun. 4:1965 doi: 10.1038/ncomms2965 (2013).

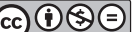

This work is licensed under a Creative Commons AttributionNonCommercial-NoDerivs 3.0 Unported License. To view a copy of this license, visit http://creativecommons.org/licenses/by-nc-nd/3.0/ 